\title{
Influencia del género en los debates electorales en España: análisis de la audiencia social en \#EIDebateDecisivo y \#L6Neldebate
}

\author{
Influence of gender in electoral debates in Spain: \\ Analysis of the social audience in \#EIDebateDecisivo \\ and \#L6Neldebate
}

\author{
Julen Orbegozo-Terradillos; Ainara Larrondo-Ureta; Jordi Morales-i-Gras
}

Cómo citar este artículo:

Orbegozo-Terradillos, Julen; Larrondo-Ureta, Ainara; Morales-i-Gras, Jordi (2020). "Influencia del género en los debates electorales en España: análisis de la audiencia social en \#EIDebateDecisivo y \#L6Neldebate". El profesional de la información, v. 29, n. 2, e290209.

https://doi.org/10.3145/epi.2020.mar.09

Artículo recibido el 15-11-2019 Aceptación definitiva: 05-02-2020

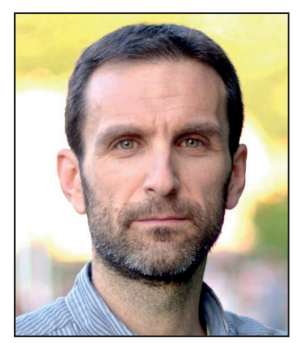

Julen Orbegozo-Terradillos $\square$ https://orcid.org/0000-0002-2959-4397

Universidad del País Vasco

Barrio Sarriena, s/n. 48940 Leioa, España julen.orbegozo@ehu.eus

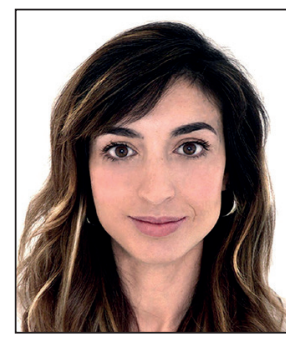

Ainara Larrondo-Ureta https://orcid.org/0000-0003-3303-4330

Universidad del País Vasco Barrio Sarriena, s/n. 48940 Leioa, España ainara.larrondo@ehu.eus

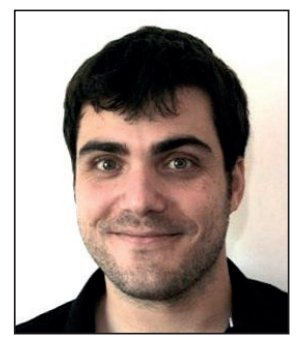

Jordi Morales-i-Gras

https://orcid.org/0000-0003-4173-3609

Universidad del País Vasco

Barrio Sarriena, s/n. 48940 Leioa, España

morales.jordi@gmail.com

\section{Resumen}

Los debates televisados constituyen uno de los elementos centrales de la comunicación política electoral. Esta investigación examina la conversación digital en Twitter surgida a raíz de dos debates durante la campaña electoral de las elecciones generales de abril de 2019 en España: \#EIDebateDecisivo de Atresmedia (entre los candidatos a la presidencia, íntegramente masculino) y \#L6Neldebate de La Sexta (íntegramente femenino). La investigación aporta una perspectiva renovada a la feminización progresiva de la política, poniendo en relación el género de las personas participantes en los debates electorales con un medio de comunicación de masas como la televisión y con la interacción de los públicos en una red social como Twitter. Se analizan 313.343 impactos de 101.510 usuarios o nodos, concluyendo que la aportación de la audiencia activa al debate público-político en el debate masculino se ubica en términos más banales y menos políticos que su equivalente femenino.

\section{Palabras clave}

Televisión; Comunicación política; Elecciones; Debates electorales; Género; Twitter; Redes sociales; Medios sociales; Política; Feminismo, Audiencias activas.

\section{Abstract}

Television debates during electoral campaigns constitute a central element within the political communication sphere. This research examines the digital conversation on Twitter on the broadcast of two television debates during the general 
elections campaign in Spain in April 2019: \#EIDebateDecisivo of Atresmedia (among male presidential candidates) and \#L6Neldebate of La Sexta (among female political candidates). The research provides a renovated perspective regarding the progressive feminisation of politics, establishing a correlation among key variables, such as the gender of the political leaders participating in the debates, the role of television as the main means during elections, and the influence of the public interaction on Twitter. Methodologically, the study pays attention to 313,343 impacts generated by 101,510 users or nodes. As pointed out in the conclusions, in the masculine debate (\#EIDebateDecisivo) the active audience's contribution to the public and political debate is defined in a more banal way, in contrast with the women's debate (\#L6Neldebate), in which a more political consciousness is perceived.

\section{Keywords}

Television; Political communication; Election; Electoral debates; Gender; Twitter; Social networks; Social media; Politics; Feminism; Active audiences.

\section{Introducción}

El debate público y el contraste de argumentos es inherente a la actividad política en todas las sociedades democráticas y ha sido definido como el intercambio ordenado y plural de argumentos y opiniones con la finalidad de hacer aportaciones que puedan influir en la res pública (Pindado; Rebollo, 2015).

En la liturgia de una campaña, el debate electoral televisado ha sido considerado uno de los géneros más importantes de la comunicación política desde los encuentros de Nixon y Kennedy en los años sesenta (Kraus, 2000; McKinney; Carlin; 2004). Según López-García et al. (2018), el debate televisado en campaña supone una escenificación y confrontación directa entre actores políticos que tratan de influir en la agenda mediática y, en última instancia, en la percepción que tiene el electorado de los problemas cotidianos. Se entiende así que este formato haya sido distinguido como el acto de comunicación de campaña con mayor audiencia y capacidad para generar grandes volúmenes de información (Luengo; García-Marín, 2009; Luengo, 2011).

Existen dos tendencias que marcan sobremanera la comunicación política contemporánea e influyen en la evolución histórica de los debates electorales televisados:

- la mediatización de la política (Sartori, 1998; Muñoz-Alonso, 1999; Castells, 2010; Espino-Sánchez, 2014);

- la tendencia a la espectacularización de lo que sucede en el espacio público (Debord, 1967; Edelman, 1988; Martínez-Pandiani, 2006).

En ambos procesos la televisión ha tenido una influencia considerable, en tanto que medio de masas principal en la vehiculización del mensaje político.

Con todo, la irrupción de internet a principios del siglo XXI y la consolidación de las redes sociales durante la segunda década del mismo han provocado transformaciones significativas en los tres actores que conforman el espacio público mediatizado: públicos, actores políticos y medios de comunicación. En este contexto, esta investigación justifica su interés al poner en valor un medio de comunicación tradicional como la televisión, a partir de una nueva relación con las audiencias de carácter político y bajo una perspectiva de género.

\subsection{Debates electorales: estado del arte y perspectiva de género}

Existen numerosas investigaciones sustentadas en distintas perspectivas sobre los debates electorales -influencia en la opinión pública, en el voto, etc.-. Recientemente han proliferado estudios relacionados con la audiencia social de los debates en las redes sociales, como Twitter (Houston et al., 2013; Elmer; Langlois, 2013; Enli; Skogerbø, 2013). Entre otros, Vergeer y Franses (2016) comparan la relación entre los temas planteados por los candidatos en el debate presidencial en los Países Bajos (2012) y los más comentados por las audiencias en las redes sociales. Burnett y Bloice (2016) analizan los enlaces aportados por los internautas durante los debates televisados en el proceso de referéndum escocés de 2014. Tremayne y Minooie (2015) ubican su estudio en el debate electoral que enfrentó a Romney y Obama en 2012 y responden desde una nueva perspectiva digital sustentada en el análisis de big data a la ya tradicional pregunta de “¿quién ganó el debate?”, a partir de las interacciones sobre dicho debate en Twitter.

La proliferación de estudios que ponen en conversación los debates electorales televisados con su correspondiente audiencia social ha abierto nuevos caminos para la investigación a partir de perspectivas renovadas. Entre éstas se echa en falta el estudio de los debates electorales desde una perspectiva de género. Esta aproximación resultaría particularmente novedosa y determinante, a tenor de la progresiva "feminización" que vive la política desde hace años. La corriente encargada del análisis político con perspectiva feminista ha definido este fenómeno al alza como inserción o integración de las mujeres en el proceso democrático a través de números y cuotas de género y sobre todo a través de ideas (Lovenduski, 2005; Kenny, 2013).

La investigación académica comenzó hace más de una década a reenfocar sus encuadres analíticos hacia el modo en el que las mujeres suponían un verdadero elemento diferenciador en la teoría y en la práctica de la política (Childs; Krook, 2006; Banwart, 2010). En esta línea se ha entendido que el aumento de mujeres en política conlleva necesariamente 
cambios sustanciales en la manera de comunicar la propia actividad política (Herrnson; Lay; Stokes, 2003; Childs, 2004), entre otros motivos, debido al uso de "ideologías lingüísticas" diferenciadas por parte de los hombres y de las mujeres (Cameron; Shaw, 2016).

De esta forma el medio de comunicación mediante el cual se transmiten los relatos políticos ha sido considerado una de las principales variables en la investigación de la feminización de la comunicación política (Norris, 1997; Bystrom et al., 2004). Junto a la publicación de análisis centrados en los medios sociales digitales (Meeks, 2013; McGregor; Mourão, 2016), se evidencia la necesidad de profundizar en campos como la televisión y sus audiencias activas. Mientras existen estudios centrados en la cobertura informativa en televisión de partidos y candidatos/as desde una perspectiva de género (Kahn; Goldenberg, 1991; Ross et al., 2013), en el caso de los debates electorales -formato por excelencia de la comunicación política en televisión-se advierte la ausencia de investigaciones bajo una perspectiva de género.

Con todo destacan hasta la fecha los estudios centrados en la política anglosajona, como el análisis de Banwart y McKinney (2005) sobre los debates electorales en las elecciones al Congreso y al Senado de Estados Unidos de 2004, o la investigación de Cameron y Shaw (2016) en torno al lenguaje y al estilo narrativo de hombres y mujeres durante los debates televisados, con motivo de las elecciones generales en Reino Unido de 2015.

En España se han analizado hasta la fecha los debates electorales en el contexto de diversas campañas, si bien ninguno de estos estudios ha puesto el matiz en la perspectiva de género. Este hecho es ciertamente lógico porque la variable género en este campo es difícil de abordar en un contexto tradicionalmente masculinizado, donde por ejemplo ha sido empíricamente imposible analizar un debate electoral a nivel nacional únicamente con candidatas a la presidencia.

En este contexto investigador, este artículo presenta un estudio pionero que pone en relación un contenido político en un medio de comunicación tradicional y su audiencia social desde una perspectiva de género. El análisis se centra en la campaña electoral de las elecciones generales del 28 de abril de 2019 en España, tomando como referencia el primer debate electoral televisado a nivel nacional en una campaña electoral a las Cortes Generales donde todas las participantes fueron mujeres (celebrado en La sexta el 13 de abril). El estudio compara este debate con el realizado entre candidatos a la presidencia, emitido por Atresmedia (23 de abril). La comparación de la conversación digital generada al calor de ambos programas sirve para obtener una radiografía de las similitudes y de las diferencias inferidas entre ambos debates.

\section{Este artículo presenta un estudio pione- ro que pone en relación un contenido político en un medio de comunicación tradicional y su audiencia social desde una perspectiva de género}

\subsection{Debates electorales en España y transición a las audiencias activas}

La historia de los debates televisados en España comenzó en 1993 con el cara a cara entre Felipe González (Partido Socialista Obrero Español, en adelante, PSOE) y José María Aznar (Partido Popular, en adelante, PP). Quince años más tarde, en 2008, y con el mismo formato, se enfrentaron José Luis Rodríguez Zapatero (PSOE) y Mariano Rajoy (PP) (Gallego-Reguera; Bernárdez-Rodal, 2017).

En la disputa televisada entre Mariano Rajoy $(P P)$ y Alfredo Pérez Rubalcaba (PSOE) en 2011 se creó por primera vez un espacio paralelo y simultáneo a la pantalla de televisión para que la audiencia comentara vía redes sociales el debate. Durante aquel año, según el III Estudio sobre redes sociales en internet (Elogia; IAB Spain, 2012) y la IV Oleada del Observatorio de Redes Sociales (The Cocktail Analysis, 2012), las redes se convirtieron en el fenómeno vertebrador de internet y en España el 75\% de los internautas era usuario de las mismas. El cara a cara entre Rubalcaba y Rajoy generó contenido más allá de la pantalla de la televisión. Las cuentas oficiales de Twitter de ambos candidatos tweetearon el evento y los televidentes convirtieron varios hashtags en trending topic, comentando el contenido de las respuestas de los protagonistas, subrayando las anécdotas o empleando elementos del debate para hacer bromas (Sánchez, 2011).

Cuatro años más tarde y en un panorama político alterado por la irrupción de nuevas formaciones como Podemos y Ciudadanos, se celebró el cara a cara entre Rajoy y Pedro Sánchez (PSOE), y el mismo año (2015) tuvo lugar el primer debate a cuatro entre Soraya Sáez de Santamaría (PP), Pedro Sánchez (PSOE), Pablo Iglesias (Podemos) y Albert Rivera (Ciudadanos). Aquel debate a cuatro abrió la puerta a otras contiendas políticas televisadas con más de dos protagonistas y supuso una evolución determinante en la manera de afrontar los debates por la configuración de la escena, la disposición de los participantes, la informalidad en la interacción de los candidatos y por haberse roto mediáticamente el modelo bipartidista (García-Marín; Calatrava; Luengo, 2018).

En los debates celebrados desde 2011 emergió además el fenómeno de las audiencias activas que generan una conversación social al interactuar con lo representado en la pantalla. Esta audiencia social (Quintas-Froufe; González-Neira, 2014) es, además de receptora, productora de contenido, lo que le dota de nuevos roles o modos de estar frente a la televisión (Orozco-Gómez,
En los debates celebrados desde 2011 emergió el fenómeno de las audiencias activas que generan una conversación social al interactuar con lo representado en la pantalla 
2014). Se transita, en otras palabras, de un medio y consumo analógico a un contexto digital, rompiendo la tradicional actitud pasiva y desmovilizadora que autoras como Hirmas (1989) otorgaban a la televisión en contextos pasados. Todo ello, como no podría ser de otra manera, influye en el diseño y la materialización de los debates electorales desde una doble perspectiva:

- el de las cadenas de televisión y sus intereses comerciales (Saavedra-Llamas; Rodríguez-Fernández, 2018);

- el de los partidos políticos y sus intereses electorales.

\section{Método}

Este estudio se enmarca en el análisis de redes sociales, una corriente analítica novedosa centrada en el descubrimiento de patrones de interacción entre actores a partir del empleo de datos masivos (big data).

Específicamente, esta investigación fija su atención en las interacciones sociales en Twitter para describir las principales similitudes y diferencias que se producen en la conversación digital en función del género masculino y femenino de los protagonistas de un debate electoral televisado. Al tomar como referencia la visión relacional de la teoría de redes, este análisis no se construye tanto a través de categorías sociales preestablecidas, sino a través de los lazos o vínculos entre actores (Lozares, 1996). Las interacciones sociales, de hecho, han constituido históricamente un objeto teórico central en las ciencias sociales (Simmel, 1908; Mead, 1934; Homans, 1958; White, 1992), aunque han permanecido infrautilizadas a nivel empírico.

Se adopta Twitter como objeto de estudio en función del potencial demostrado de esta red social para conectar a la ciudadanía con los líderes políticos (Tremayne; Minooie, 2015). Desde un punto de vista general, Twitter se ha posicionado como la red principal en el universo de la audiencia social por su fácil uso, por permitir el comentario en tiempo real, por fomentar interacciones con otros usuarios, con el programa, con los protagonistas, etc. (Castelló-Martínez, 2013).

Además de ofrecer una descripción general de cada conversación digital, el estudio identifica los usuarios que participaron en ambos debates y analiza su comportamiento en contraste con quienes participaron solamente en el debate más masivo (el de hombres). A esto lo denominaremos "correspondencia de perfiles entre clústeres".

Los debates electorales televisados que sirven para elaborar este estudio y la información referente a los mismos se determinan en la tabla 1.

Tabla 1. Información sobre los debates monitorizados

\begin{tabular}{|c|c|c|c|c|c|}
\hline $\begin{array}{c}\text { Nombre debate / } \\
\text { programa }\end{array}$ & Canal & Participantes & $\begin{array}{l}\text { Fecha de } \\
\text { emisión }\end{array}$ & URL & Hashtag \\
\hline $\begin{array}{l}\text { Debate Atresmedia } \\
\text { (El debate decisivo) }\end{array}$ & $\begin{array}{l}\text { Atresmedia } \\
\text { (Antena } 3 \text { / La sexta) }\end{array}$ & $\begin{array}{l}\text { Pedro Sánchez (PSOE) } \\
\text { Pablo Casado (PP) } \\
\text { Pablo Iglesias (Unidas Podemos) } \\
\text { Albert Rivera (Ciudadanos) }\end{array}$ & $23 / 4 / 2019$ & https://cutt.ly/lei4IC1 & \#EIDebateDecisivo \\
\hline La sexta noche & La sexta & $\begin{array}{l}\text { María Jesús Montero (PSOE) } \\
\text { Cayetana Álvarez de Toledo (PP) } \\
\text { Irene Montero (Unidas Podemos) } \\
\text { Inés Arrimadas (Ciudadanos) }\end{array}$ & $13 / 4 / 2019$ & https://cutt.ly/zei4Hop & \#L6Neldebate \\
\hline
\end{tabular}

Según las hipótesis que han guiado este estudio, los usuarios presentes en el debate femenino tenderán a participar también en el masculino, numéricamente más masivo (H1). Asimismo, los usuarios presentes en el debate femenino que participen en el masculino lo harán en clusters en los que predominarán los contenidos políticos en vez de los memes y el humor (i.e. contenidos banales) (H2). Por último, este análisis ha considerado que la conversación alrededor del debate masculino tenderá más hacia los hiperliderazgos, mientras que el debate femenino suscitará mayor horizontalidad en los liderazgos (H3).

Para la conformación de la muestra del estudio se llevan a cabo diferentes procedimientos. De una parte, se captura el corpus de interacciones a partir de la monitorización de los correspondientes hashtags (tabla 1) entre las 19:00 y las 23:59 horas de los días correspondientes al debate, obteniendo datos durante el programa, pero también en los momentos previos y posteriores. Para ello se emplea la aplicación The digital methods initiative-Twitter capture and analysis toolset (DMI-TCAT), creada por la Universidad de Amsterdam (Borra; Rieder, 2014).

Posteriormente se sintetizan los grafos de red generados para cada debate a través del programa DMI-TCAT. En estos grafos, cada actor o nodo representa un participante en la conversación, y cada conexión una interacción de tipo mención en Twitter: un retweet, una respuesta directa o una interpelación dentro de un tweet regular. Dichas redes se insertan en el software Gephi (Bastian; Heimann; Jacomy, 2009) y se interpretan como una red dirigida y ponderada. Para extraer y calcular las métricas de la red se emplearon los programas Gephi y Pajek (Batagelj; Mrvar, 1998). Específicamente, para la evaluación empírica y la validación hipotética se tienen en cuenta los siguientes indicadores:

- Impactos totales: número de publicaciones capturadas, ya sean tweets o retweets.

- Nodos: número de usuarios únicos que han participado en la conversación, quedando excluidos los que no han mencionado ni han sido mencionados por ningún otro usuario. 
- Media de impactos por usuario: número de publicaciones que cada autor ha efectuado de media en la conversación.

- Aristas: número de interacciones únicas establecidas entre usuarios. Cada arista tiene un peso o ponderación asociada al volumen de menciones que representa: cuando una arista represente una sola mención tendrá de peso o ponderación 1, cuando represente 20 menciones tendrá de peso o ponderación 20.

- Grado medio ponderado: número de usuarios a los que cada nodo está asociado de media.

- Distancia máxima: número de aristas o "saltos" entre nodos que separan los dos nodos alcanzables más alejados en la red.

- Distancia media: número de aristas o "saltos" entre nodos que separan los nodos alcanzables en la red de media.

- Centralización de grado de entrada: métrica no presente en Gephi y calculada mediante Pajek que proporciona una cifra entre 0 y 1 en la que 0 indica descentralización total (i.e. todos los nodos reciben menciones por parte de la misma cantidad de usuarios únicos) y 1 indica centralización total (i.e. un solo nodo recibe menciones por parte del resto de usuarios únicos). El algoritmo está basado en la fórmula de Freeman (1978) para la centralización de grado.

- Modularidad: valor "Q" asociado a la calidad de la partición de la red en distintos clusters o comunidades producida por el algoritmo para la identificación de comunidades de Gephi (Blondel et al., 2008). Este algoritmo da cuenta de la proporción de enlaces que quedan capturados por los clusters generados algorítmicamente. Según la fórmula general para la modularidad definida por Newman y Girvan (2004) valores iguales o superiores a 0,3 indican significatividad matemática.

- Número de comunidades: número total de clusters identificados por el algoritmo anterior. Entre paréntesis (en la tabla 2) destacamos el número de clusters que agrupan a más del $2 \%$ de los nodos o usuarios.

Los cálculos anteriores proporcionan información sobre el conjunto de la red y generan una serie de indicadores que operan a nivel de cada nodo de la red. Para esta investigación el análisis se centra en los siguientes indicadores nodales:

- Grado de entrada ponderado: suma de menciones que recibe un usuario en la red.

- Clúster o comunidad de pertenencia: variable categórica expresada ordinalmente (i.e. comunidad "1", comunidad "2", etc.) que da cuenta del clúster en el que ha sido ubicado cada nodo por el algoritmo de identificación comunitaria.

El procedimiento metodológico descrito permite obtener la información necesaria para describir la red, sintetizar los grafos, ver los contenidos y los actores más exitosos de las comunidades que emergen en la conversación digital, analizar la correlación y correspondencia de los participantes entre los clusters de las diferentes emisiones y obtener información en torno al tipo de debate surgido en Twitter a raíz de los debates o algunas referencias a la calidad del mismo.

\section{Resultados}

\subsection{Aproximación general a la red}

Una aproximación general a la red permite observar una primera diferencia en la comparación de ambos clusters: el volumen de impactos totales y de usuarios interpelados en el debate protagonizado por hombres es ostensiblemente mayor (tabla 2). Estos datos son relativamente previsibles, teniendo en cuenta dos factores:

- en el Debate Atresmedia actuaron los candidatos presidenciables. En cambio en el de La sexta noche los partidos eligieron a sus representantes femeninas independientemente del puesto que ocupaban en las listas electorales;

- la empresa de comunicación Atresmedia creó un evento mucho mayor en torno al debate protagonizado por los candidatos.

Con todo, quien participa en el programa de La sexta noche lo hace con una frecuencia de interacción mayor que en el programa protagonizado íntegramente por hombres. De media, en la conversación de \#ElDebateDecisivo cada perfil o usuario interviene tres veces. Este número es de cuatro interacciones de media por actor en el debate de \#L6Neldebate de la La sexta noche.

En relación con la red obtenida apoyada en la teoría de grafos, las figuras 1 y 2 proporcionan información sobre la morfología general de las conversaciones digitales de ambos debates. Del debate masculino se derivan 11 comunidades que concentran un número mayor de las interacciones totales del cluster (más del 2\%) (tabla 3). Por su parte, en el debate femenino son 7 las comunidades resultantes (tabla 4) bajo Tabla 2. Tabla comparativa de las métricas extraída de las dos conversaciones analizadas los mismos criterios.

\begin{tabular}{|l|c|c|}
\cline { 2 - 3 } \multicolumn{1}{c|}{} & \#EIDebateDecisivo & \#L6Neldebate \\
\hline Impactos totales & 273.238 & 40.105 \\
\hline Nodos o usuarios interpelados & 92.052 & 9.458 \\
\hline Media de impactos por usuario & 2,97 & 4,24 \\
\hline Aristas (impactos únicos) & 226.336 & 31.107 \\
\hline Grado medio ponderado & 3,09 & 5,27 \\
\hline Distancia máxima & 37 & 15 \\
\hline Distancia media & 12,91 & 6,18 \\
\hline Centralización de Grado de entrada & 0,07 & 0,35 \\
\hline Modularidad & 0,60 & 0,47 \\
\hline Número de comunidades & $1996(11>2 \%)$ & $84(7>2 \%)$ \\
\hline
\end{tabular}


Tabla 3. Breve descripción de las comunidades derivadas de \#EIDebateDecisivo

\begin{tabular}{|c|c|c|}
\hline Comunidad nº & Líder (indegree) & Tema del cluster \\
\hline 1 & @aqualayx & Humor sobre el debate \\
\hline 2 & @srfortfast & Humor sobre el debate \\
\hline 3 & @pablo_iglesias_ & Apoyo a Unidas Podemos \\
\hline 4 & @pablocasado_ & Apoyo al $P P$ \\
\hline 5 & @gerardotc & Humor, crítica y apoyo a Podemos \\
\hline 6 & @albert_rivera & Apoyo y críticas a Ciudadanos \\
\hline 7 & @sanchezcastejon & Apoyo al PSOE \\
\hline 8 & @psoe & Comunicación corporativa de todos los partidos (a) \\
\hline 9 & @el_pais & Medios de comunicación tradicionales y digitales (b) \\
\hline 10 & @lavecinarubia & Humor sobre el debate \\
\hline 11 & @jordisan & Humor sobre el debate \\
\hline
\end{tabular}

Tabla 4. Breve descripción de las comunidades derivadas de \#LSNelDebate

\begin{tabular}{|c|l|l|}
\hline Comunidad $\mathbf{n}^{\mathbf{0}}$ & \multicolumn{1}{|c|}{ Líder (indegree) } & \multicolumn{1}{c|}{ Tema del cluster } \\
\hline 1 & @irene_montero_ & Apoyo a Podemos \\
\hline 2 & @ahorapodemos & Apoyo a Podemos \\
\hline 3 & @inesarrimadas & Apoyo a Ciudadanos \\
\hline 4 & @protestona1 & Apoyo a Podemos \\
\hline 5 & @sextanochetv & Apoyo a PSOE \\
\hline 6 & @populares & Apoyo a PP \\
\hline 7 & @anggomz & Apoyo a Podemos \\
\hline
\end{tabular}

Los grafos derivados de cada debate sintetizan así cada conversación digital que detallamos en los siguientes apartados.

\section{\#EIDebateDecisivo (figura 1)}

Se trata de una red relativamente compacta, donde sobresalen las comunidades creadas en torno a contenidos o interacciones basadas en el humor, memes, chistes y burlas (alrededor de un tercio de la conversación gira en torno a estos temas). De las 11 comunidades destacadas, 5 contienen estos temas, tendiendo a situarse hacia la periferia del grafo. La

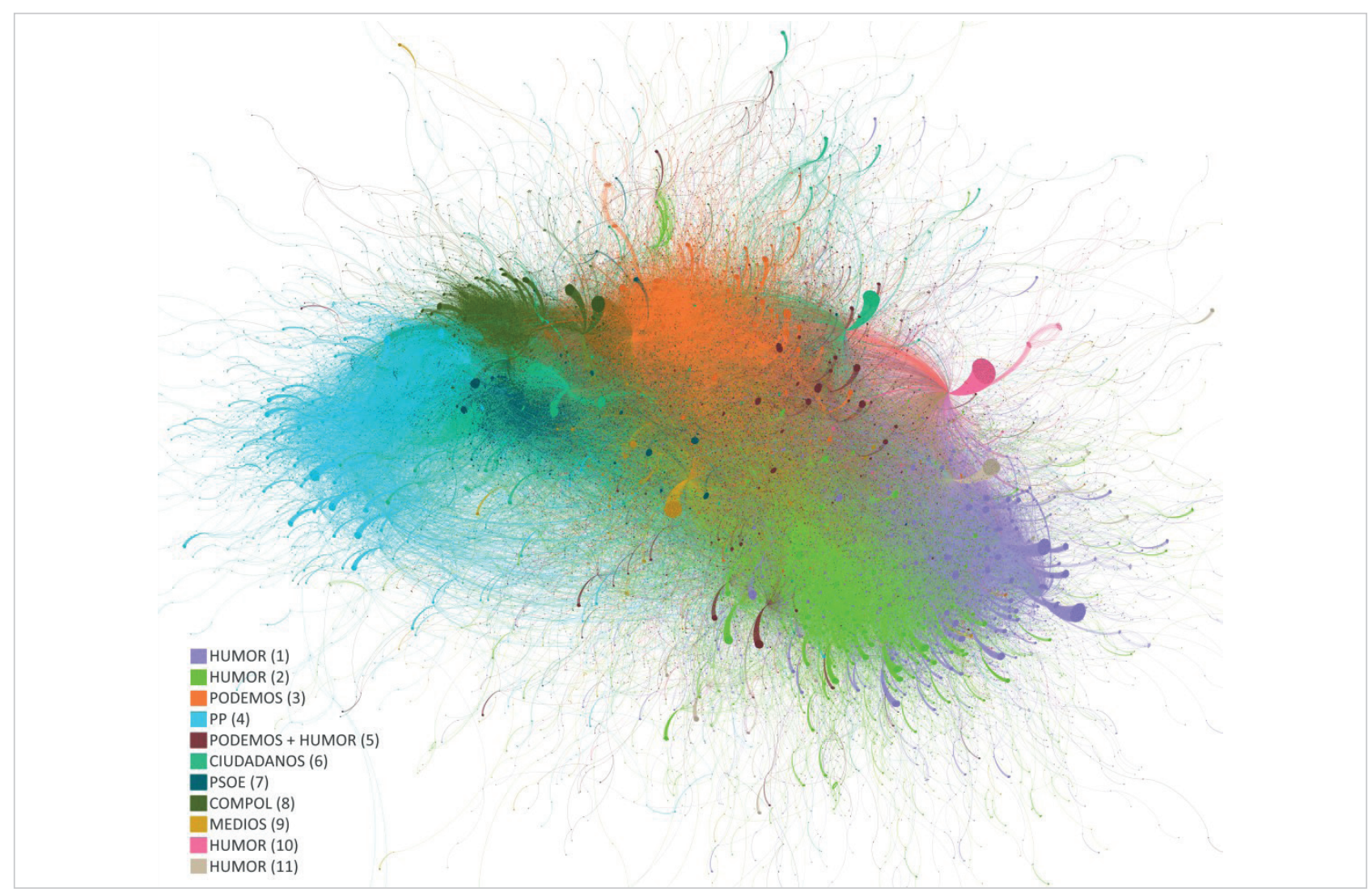

Figura 1. Visualización de red de \#DebateAtresmedia 
comunidad que interviene como intermediaria entre el citado humor y el resto del grafo es el clúster de apoyo a Unidas Podemos, liderado por el máximo responsable de la formación, Pablo Iglesias.

Unidas Podemos es entre los cuatro partidos el que más interacciones suscita, con una posición de mediador en la conversación. La comunidad erigida en torno al Partido Popular le sigue de cerca, aunque su posición en el grafo es algo más aislada, y definitivamente más distanciada del código humorístico que rodea a la comunidad de Unidas Podemos. PSOE y Ciudadanos constituyen su propia congregación en torno a su marca política, aunque con una presencia en la conversación mucho menor que los otros dos partidos.

Al margen del humorismo y de los clusters relacionados con los partidos, germinan otros dos grupos:

- los medios de comunicación tradicionales y digitales que hacen un seguimiento informativo del debate;

- agrupación que congrega a la comunicación corporativa oficial de los partidos políticos.

Mención especial en este apartado a la presencia del ingenio y el humor en todas sus variantes: socarronería, humor ácido, ironía, burlas y memes. La tipología del humor empleado es diversa, pero una somera observación nos lleva a clasificar estas interacciones en dos grandes fracciones:

- los tweets que emplean el humor en torno a algún contenido explicitado en el debate (figura 2);

- las interacciones mayoritarias erigidas en torno a creaciones ficticias, fotomontajes, apropiaciones o parodias sin relación alguna con el contenido del debate (figura 3).

Por último, destacan en esta red las interacciones dedicadas a la caracterización de los cuatro líderes políticos. Constituyen mensajes personalizados en torno a los candidatos, bien sean de sus propios seguidores ensalzando sus bondades, o bien sean las de comunidades en torno a partidos políticos que lanzan mensajes para desacreditar o difamar al contrario.

\section{\#L6Neldebate (figura 5)}

Se trata de una red con menor volumen de interacciones, pero con nodos más activos (i.e. cada usuario ha publicado una media de 4,24 tweets o retweets, frente a los 2,97 de la otra red) y con mayor grado de conectividad: cada nodo de la red está asociado a una media de 5,3 nodos más (i.e., grado medio ponderado), mientras que en el debate masculino esta cifra es de 3,1.
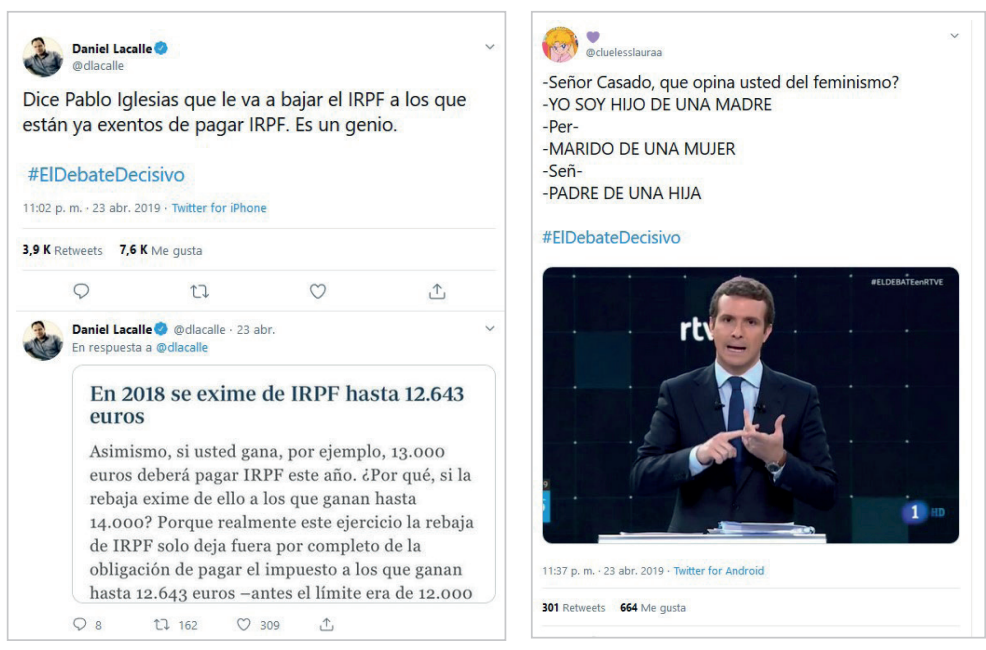

Figura 2. Serie de tweets de contenido irónico-humorístico relacionado con contenido político del debate
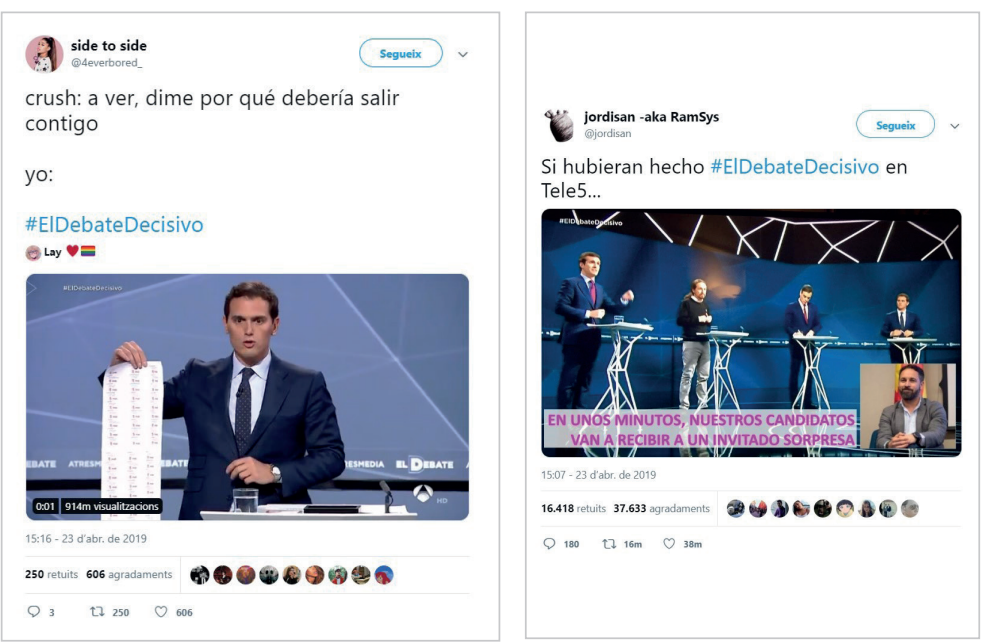

Figura 3. Serie de tweets humorísticos exitosos no relacionados directamente con el contenido político del debate

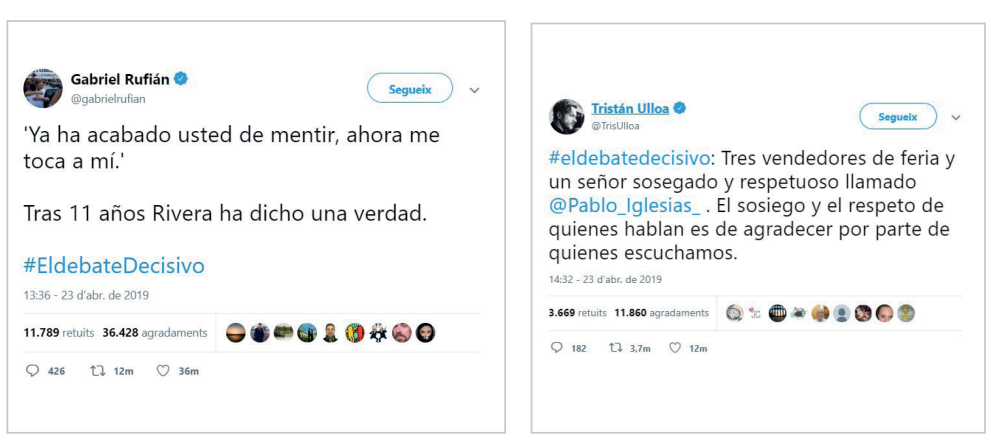

Figura 4. Serie de tweets destacados que caracterizan al líder propio o al contrario político por parte de la audiencia social, centrada principalmente en utilizar los personajes, los argumentos y el contexto para hacer humor, mofas o chistes 
Siendo así, tweetea y es interpelado un número menor de perfiles y personas. Con todo, los usuarios tienen una mayor probabilidad de acceder a los contenidos de la red. Asimismo el número de pasos que hay entre los nodos más alejados (i.e., distancia máxima) es la mitad que en el debate masculino y sucede lo mismo con la distancia media entre los nodos para conectarse entre sí (i.e., distancia media). Este hecho denota que esta es una red más cohesionada, donde la información fluye más ágilmente y es más factible que un mensaje generado en cualquier punto de la red llegue a cualquier otro punto de la misma.

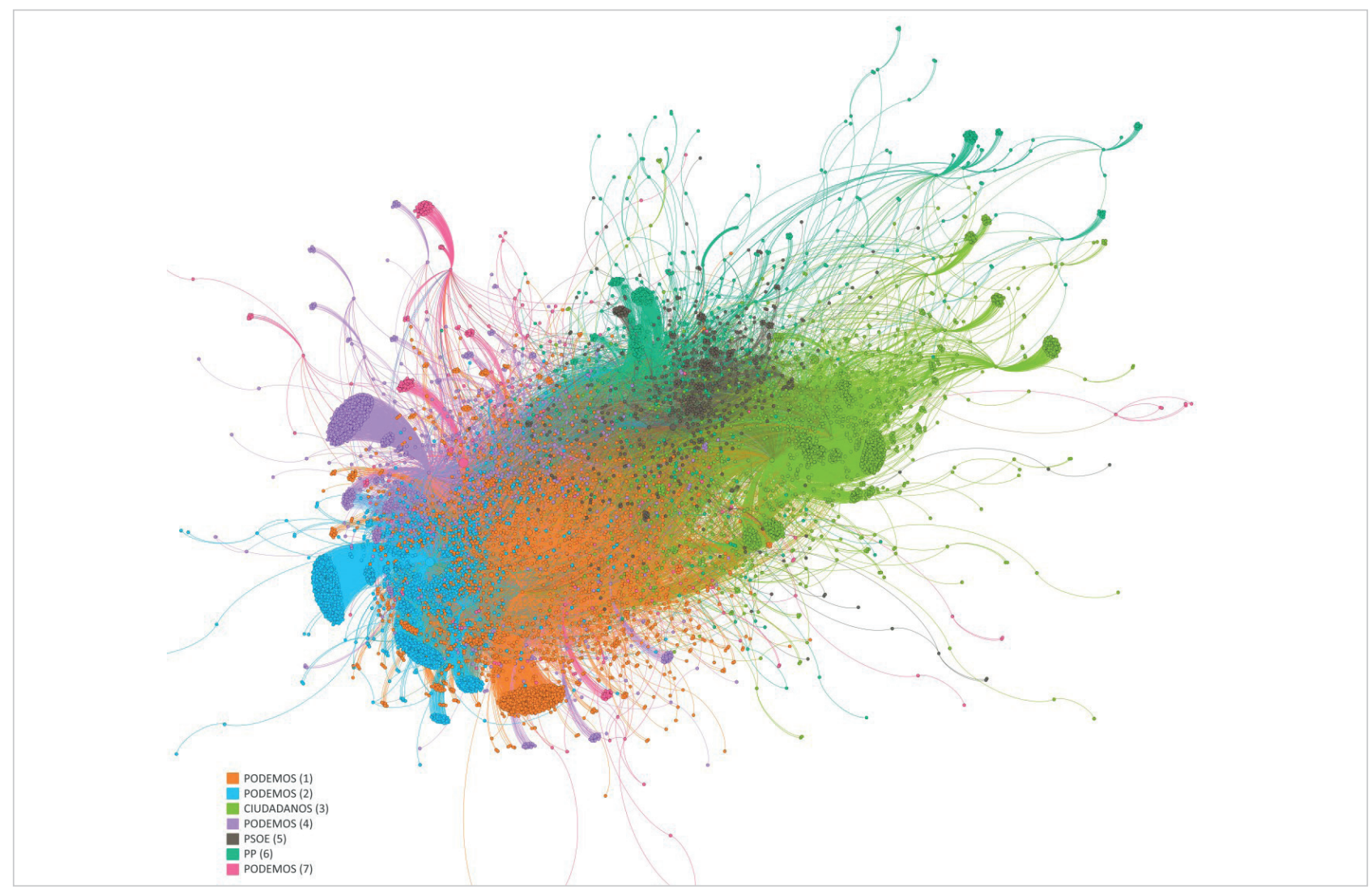

Figura 5. Visualización de red \#L6Neldebate

La observación del grafo resultante manifiesta que la conversación gira mayormente en torno a los contenidos creados por la candidata (Irene Montero) y la marca de Unidas Podemos. Las interpelaciones a esta formación constituyen el eje central de 4 de las 7 comunidades. Ello sugiere que el electorado afín a Unidas Podemos está más movilizado en la conversación objeto de estudio. También Ciudadanos obtiene cierto protagonismo, mientras que el resto de los partidos son prácticamente testimoniales.

En este caso y marcando una enorme distancia con el debate masculino, el humor es minoritario en todos los clusters. De hecho las comunidades originadas para apoyar a cada partido persiguen caracterizar o aupar a sus propias candidatas. Los contenidos centrales versan sobre el contenido político de declaraciones de las participantes, en muchos
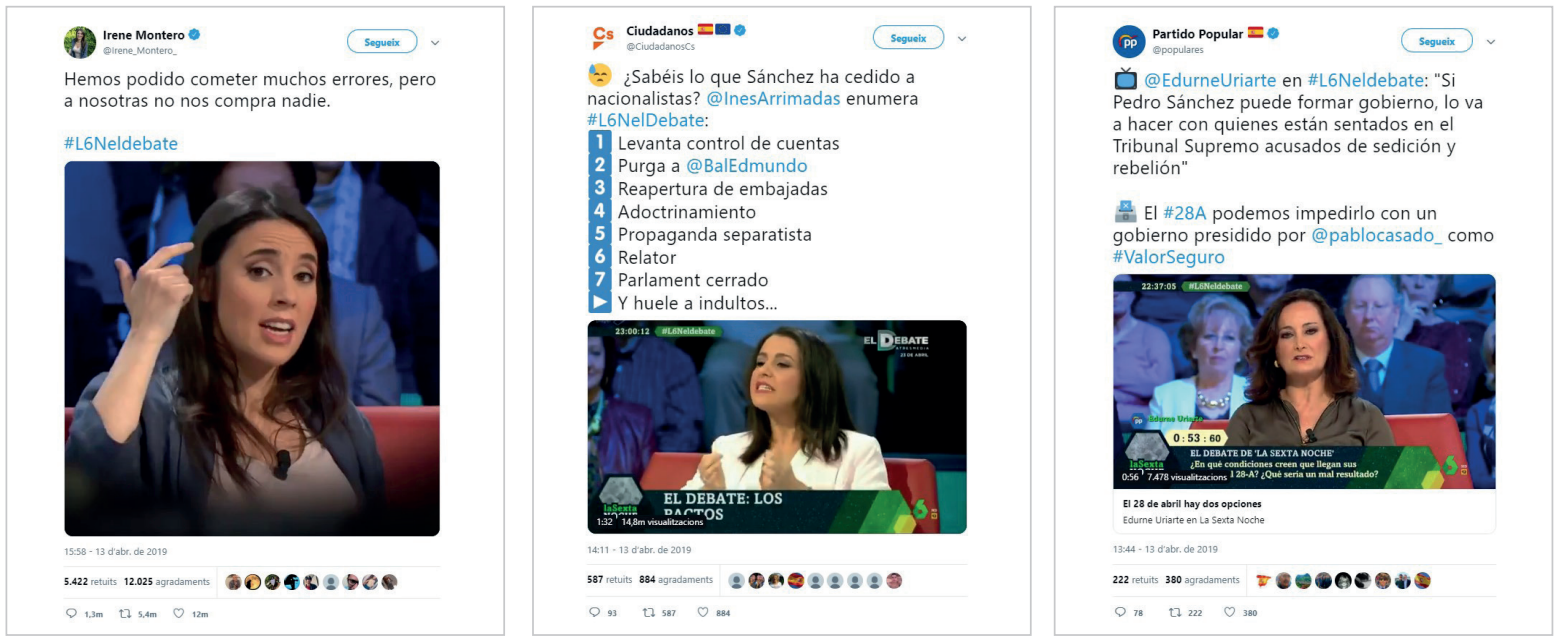

Figura 6. Serie de tweets más populares de los clusters 1,3 y 6 
casos expandidos por las cuentas oficiales de los propios partidos o de las candidatas políticas. El tipo de mensaje que caracteriza la red es, por así decirlo, corporativo y alineado con los estándares del marketing y la comunicación política. Se debate sobre los mismos contenidos o declaraciones que suceden en el programa, en vez de recurrir a recursos exógenos.

\section{Casi la mitad de los internautas que par- ticiparon en el debate femenino lo hicie- ron en el debate masculino}

\subsection{Correspondencia de clusters (figura 7)}

A través de la aplicación del algoritmo Louvain multilevel se identifican comunidades y se detectan los perfiles top in-degree o top betwenness en cada cluster. De esta manera se especifica en cierta forma la propia existencia de la comunidad en base a sus liderazgos. En este paso se trata de resolver si la gente o los perfiles que han sido clusterizados por los algoritmos en el debate de mujeres también han participado en la conversación digital del debate masculino. Asimismo en caso afirmativo, a qué grupo o cluster se han inscrito. De esta forma observamos si aquellos perfiles que repiten participación en uno y otro debate se agrupan en determinados clusters.

La correspondencia de los perfiles en diferentes clusters nos indica precisamente qué comunidades han conseguido atraer a esa porción de audiencia activa que ha repetido visionado y participación en los dos debates.

Observamos que el $44,58 \%$ de los usuarios presentes en el debate femenino están presentes en el masculino. Es decir, casi la mitad de los internautas que participaron en el debate femenino lo hicieron en el debate masculino (H1) y solamente el 13,93\% de los participantes del debate masculino que estuvieron presentes en el femenino formaron parte de clusters humorísticos y sarcásticos (H2). La segunda hipótesis de partida queda fuertemente validada, porque la mayoría de los internautas que repiten participación lo hacen en clusters eminentemente políticos y no humorísticos que son precisamente los más exitosos, con diferencia, en el caso masculino. El 39,86\% de los usuarios que repiten participación, por ejemplo, se implicaron en uno de los clusters de apoyo a Unidas Podemos.

Según los datos obtenidos, el clúster de \#EIDebateDecisivo que apoya a Unidas Podemos congrega a un gran número de internautas que también participan en \#L6Neldebate en, precisamente, los clusters de apoyo al mismo partido. Es decir, el partido de izquierdas y su líder generan interés y conversación digital entre la audiencia social. Por otro lado, el resto de partidos ( $P S O E, P P$ y Ciudadanos) congregan un número similar de perfiles repetidos, pero en un grado mucho menor que el de Unidas Podemos.

El clúster que agrupa la comunicación corporativa de los partidos ofrece un dato similar al de PSOE, PP y Ciudadanos, es decir, no consigue constituir un eje prioritario para que la gente participe en la conversación digital de ambos debates a través de la comunicación corporativa. Algo similar sucede con el clúster de los medios de comunicación.

Por último, la Centralización de grado de entrada ponderada es mayor en el caso femenino que en el masculino, con un dato del 0,35 (35\%) y 0,07 (7\%), respectivamente (H3). Es decir, contrariamente a lo que se podía prever en un principio,

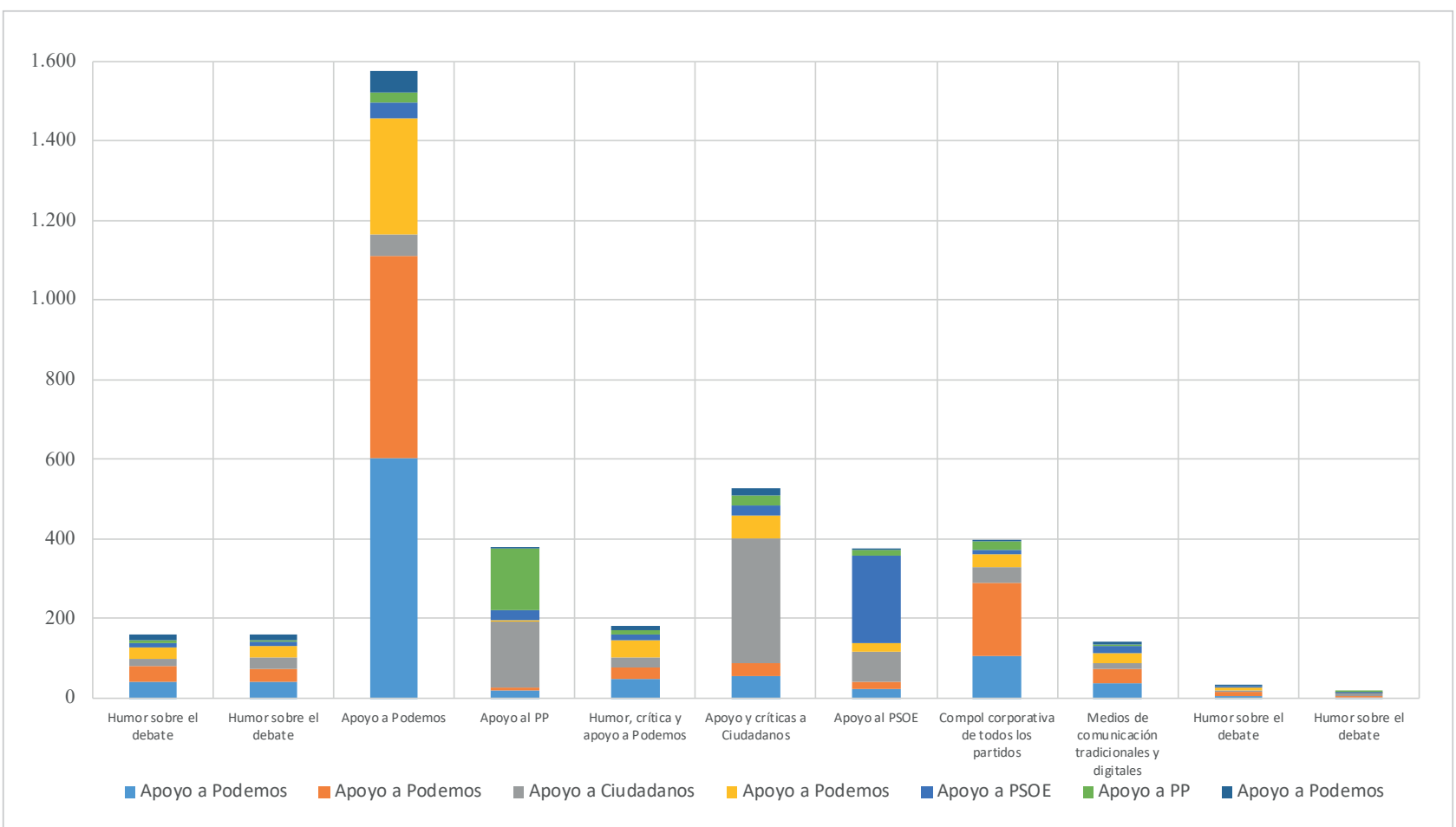

Figura 7. Visualización gráfica de la correspondencia entre clusters de los dos debates analizados 
el grupo de nodos que reciben muchas menciones es menor en \#L6Neldebate (mayor tendencia a la verticalidad de las interacciones), situándonos en un escenario de mayor horizontalidad dialógica en el debate masculino.

Todo indica que este hecho se debe a la multiplicación de actores mediáticos que participan de la conversación y a la diversificación temática correspondiente. De hecho, la importancia mediática atribuida a \#EIDebateDecisivo y la participación masiva de actores en Twitter conllevó a que personajes reconocidos en el espacio público no presentes en el debate obtuvieran gran impacto con sus interacciones: Santiago Abascal (Vox), Gabriel Rufián (Esquerra Republicana de Catalunya), Alberto Garzón (Unidas Podemos) o Ana Pastor (La sexta). A ellos se les sumaron internautas de menor resonancia pública, pero con gran notoriedad en este evento como @lavecinarubia o @aqualyx. Estos usuarios cosechan grandes cantidades de retweets utilizando el hashtag del programa, desdibujando así los hiperliderazgos de los candidatos en antena.

\section{Discusión y conclusiones}

Este trabajo utiliza las redes relacionales surgidas a raíz de dos debates políticos televisados y sus respectivas conversaciones digitales para trazar una línea de unión entre la feminización de la política, el medio de masas predilecto tradicionalmente por la comunicación política (la televisión), y la audiencia social interactiva como gran particularidad de la sociedad actual. El estudio consigue así poner de manifiesto de qué manera

"las redes son formas de interacción social, definida como un intercambio dinámico entre personas, grupos e instituciones en contextos de complejidad" (Dabas; Najmanovich, 1996).

Cuanto mayor seguimiento tiene un producto televisivo como por ejemplo un debate electoral, tenderá a ser mayor la audiencia social que genera. Extrapolado este hecho a nuestro objeto de estudio, el debate realizado con hombres candidatos congregó a más audiencia analógica frente a la televisión ( 9.477 .000 telespectadores) que el debate de mujeres candidatas (1.027.000), de ahí que resulte lógico que la conversación digital sea más voluminosa en el debate masculino que en el femenino.

De esta forma, según confirma este estudio, aquellos internautas que repitieron participación en la conversación digital en Twitter de ambos debates, el femenino (\#L6Neldebate) y el masculino (\#EIDebateDecisivo), no lo hicieron congregándose en torno a comunidades donde el contenido principal fuera el humor banal, el chiste, la burla o la ridiculización del contrario. Sólo una pequeña cantidad de internautas pasaron del debate femenino al masculino para participar en los clusters humorísticos. De hecho, se intuye que el debate entre mujeres, menos masificado en su audiencia interactiva por motivos varios, tiene un nicho propio de internautas activos que potencia un debate de mayor calidad en términos democráticos.

Ambos debates tienen audiencias relativamente diferenciadas cuantitativa y cualitativamente. Por otro lado, las mujeres candidatas siguen transitando por un camino sidestreaming o paralelo, protagonizando en La sexta noche un debate donde el actor estándar de la conversación digital participa buscando, si cabe, un producto político en un medio de comunicación determinado. A la inversa, en el debate masculino convertido en mainstream, muchos de los internautas interpretan que los políticos intervienen como materia prima moldeable a sus necesidades de espectáculo, diversión y cierta trivialidad.

Así, la masificación del debate de Atresmedia (\#EIDebateDecisivo) conlleva una suerte de banalización del discurso político por parte de la audiencia social, centrada principalmente en utilizar a los personajes, los argumentos y el contexto de la escena para hacer humor, mofas o chistes. Este hecho no es de ninguna forma una circunstancia que deba obviar nuestro sistema democrático. La tendencia a la espectacularización de lo que sucede en el espacio público y político mediatizado abre la puerta a nuevos consumidores de lo político que buscan una vía de escape ante el descrédito general de la política, pero ello no conlleva que la calidad del debate público mejore en términos democráticos.

Efectivamente, el humor, que tanta presencia tiene en todas sus variantes en este estudio, es uno de los caminos más cortos y plácidos para persuadir a alguien. El filósofo francés Bergson (1940) explicaba la utilidad del humor para mantener el cerebro de alguien activo mientras recibe un mensaje y destacaba su potencial como medio socializador y elemento de cohesión grupal. Sin embargo, cabe pensar que en el caso analizado el humor no se emplea con fines tácticos ni estratégicos en beneficio de fines democráticos; se emplea más bien dentro de la lógica comercial de un medio tradicional como la televisión, en constante evolución para reinventarse en el nuevo ecosistema mediático.

Sistema político y televisión, ambos se benefician de esta dualidad. Sin embargo, en el debate más masivo donde interactúan los candidatos masculinos, la televisión y sus fines se "comen” a lo político, incluso erosionándolo. En el de mujeres por su parte, la audiencia social se relaciona y dialoga entre más gente, emplea otros recursos retóricos, moviéndose por otros derroteros más acordes con los objetivos del sistema democrático, fomentando el debate público a través de Twitter, que demuestra su carácter de espacio de igualdad de oportunidades a la hora de acceder a la opinión pública. 
En el tridente que reúne a los elementos de la comunicación pública y política (Mazzoleni, 2010) el sistema político avanza hacia una relativa sumisión respecto a los otros dos actores más empoderados en el nuevo escenario comunicativo, el ecosistema mediático y el público ciudadano. Bajo esa premisa, la investigación realizada permite entrever cierta instrumentalización del debate político por parte de la televisión para sus fines comerciales en un contexto interactivo.

\section{Referencias}

Banwart, Mary-Christine (2010). "Gender and candidate communication: Effects of stereotypes in the 2008 election". American behavioral scientist, v. 54, n. 3, pp. 265-283.

https://doi.org/10.1177/0002764210381702

Banwart, Mary-Christine; McKinney, Mitchell S. (2005). "A gendered influence in campaign debates? Analysis of mixedgender United States Senate and Gubernatorial debates”. Communication studies, v. 56, n. 4, pp. 353-373. https://doi.org/10.1080/10510970500319443

Bastian, Mathieu; Heimann, Sebastien; Jacomy, Mathieu (2009). “Gephi: An open source software for exploring and manipulating networks". In: International AAAl Conference on weblogs and social media.

Batagelj, Vladimir; Mrvar, Andrej (1998). "Pajek, program for large network analysis". Connections, v. 21, n. 2, pp. $47-57$. http://vlado.fmf.uni-lj.si/pub/networks../doc/pajek.pdf

Bergson, Henri (1940). La risa. Madrid: Espasa Calpe. ISBN: 9788423915347

Blondel, Vincent D.; Guillaume, Jean-Loup; Lambiotte, Renaud; Lefebvre, Etienne (2008). “Fast unfolding of communities in large networks". Journal of statistical mechanics: Theory and experiment, v. 8, n. 10, P10008. https://iopscience.iop.org/article/10.1088/1742-5468/2008/10/P10008/pdf

Borra, Erik; Rieder, Bernhart (2014) "Programmed method: Developing a toolset for capturing and analyzing tweets". Aslib journal of information management, v. 66, n. 3, pp. 262-278.

https://doi.org/10.1108/AJIM-09-2013-0094

Burnett, Simon; Bloice, Lyndsay (2016). "Linking for influence: Twitter linked content in the Scottish referendum televised debates". Journal of information science, v. 42, n. 3, pp. 396-409.

https://doi.org/10.1177/0165551515624355

Bystrom, Dianne G.; Robertson, Terry; Banwart, Mary-Christine; Kaid, Lynda-Lee (2005). Gender and candidate communication: Videostyle, webstyle, newstyle. New York: Routledge. ISBN: 9780203323137 https://doi.org/10.4324/9780203323137

Cameron, Deborah; Shaw, Silvia (2016). Gender, power and political speech. Women and language in the 2015 UK general election. London: Palgrave Macmillan. ISBN: 9781137587527

Castelló-Martínez, Araceli (2013). "El uso de hashtags en Twitter por parte de los programas de televisión españoles". En: Segado, Francisco (dir.). Actas del I Congreso internacional comunicación y sociedad. Logroño: Universidad Internacional de La Rioja. ISBN: 9788415626428

http://rua.ua.es/dspace/handle/10045/67420

Castells, Manuel (2010). Comunicación y poder. Madrid: Alianza Editorial. ISBN: 9788420684994

Childs, Sarah (2004). "A feminised style of politics? Women MPs in the House of Commons". The British journal of politics and international relations, v. 6, n. 1, pp. 3-19.

https://doi.org/10.1111/j.1467-856X.2004.00124.x

Childs, Sarah; Krook, Mona-Lena (2006). "Gender and politics: The state of the art". Politics, v. 26, n. 1, pp. 18-28. https://doi.org/10.1111/j.1467-9256.2006.00247.x

Dabas, Elina; Najmanovich, Denise (1996). Redes, el lenguaje de los vínculos: hacia la reconstrucción y el fortalecimiento de la sociedad civil. Buenos Aires: Paidós. ISBN: 9789501270259

Debord, Guy (1967). La société du spectacle. Detroit: Black \& Red. ISBN: 9782072779404

Edelman, Murray (1988). Constructing the political spectacle. Chicago: The University of Chicago Press. ISBN: 97802261 83994

Elmer, Greg; Langlois, Ganaele (2013). "Networked campaigns: Traffic tags and cross platform analysis on the web". Information polity, v. 18, n. 1, pp. 43-56.

https://doi.org/10.3233/IP-2011-0244 
Elogia, IAB Spain (2012). III Estudio sobre redes sociales en internet.

https://es.slideshare.net/IAB_Spain/iii-estudio-sobre-redes-sociales-en-internet

Enli, Gunn-Sara; Skogerb $\varnothing$, Eli (2013). "Personalized campaigns in party-centred politics". Information, communication \& society, v. 16, n. 5, pp. 757-774.

https://doi.org/10.1080/1369118X.2013.782330

Espino-Sánchez, Germán (2014). "La política en internet, ¿de la mediatización a la convergencia digital?”. Convergencia, v. 21, n. 65 , pp. 39-63.

http://www.scielo.org.mx/scielo.php?script=sci_arttext\&pid=S1405-14352014000200002

Freeman, Linton C. (1978). “Centrality in social networks: Conceptual clarification”. Social networks, v. 1, n. 3, pp. $215-239$.

Gallego-Reguera, María; Bernárdez-Rodal, Asunción (2017). “Influencia y repercusión mediática de los debates 'cara a cara' celebrados ante las elecciones generales de 2008 en España”. Vivat Academia, n. 141, pp. 139-154.

http://doi.org/10.15178/va.2017.141.139-154

García-Marín, Javier; Calatrava, Adolfo; Luengo, Óscar G. (2018). “Debates electorales y conflicto. Un análisis con máquinas de soporte virtual (SVM) de la cobertura mediática de los debates en España desde 2008". El profesional de la información, v. 27, n. 3, p. 624-632.

https://doi.org/10.3145/epi.2018.may.15

Herrnson, Paul S.; Lay, J. Celeste; Stokes, Atiya-Kai (2003). “Women running 'as women': Candidate gender, campaign issues, and voter-targeting strategies". Journal of politics, v. 65, n. 1, pp. 244-255.

https://doi.org/10.1111/1468-2508.t01-1-00013

Hirmas, María-Eugenia (1989). “La campaña electoral en la era de la TV”. Nueva sociedad, n. 99, pp. 32-39.

https://www.nuso.org/media/articles/downloads/1713_1.pdf

Homans, George C. (1958). "Social behavior as exchange”. American journal of sociology, v. 63, n. 6, pp. 597-606.

Houston, J. Brian; McKinney, Mitchell S.; Hawthorne, Joshua; Spialek, Matthew L. (2013). "Frequency of tweeting during presidential debates: Effect on debate attitudes and knowledge". Communication studies, v. 64, n. 5, pp. 548-560. https://doi.org/10.1080/10510974.2013.832693

Kahn, Kim-Fridkin; Goldenberg, Edie N. (1991). "Women candidates in the news: An examination of gender differences in US Senate campaign coverage". Public opinion quarterly, v. 55, n. 2, pp. 180-199.

Kenny, Meryl (2013). Gender and political recruitment. Theorizing institutional change. London: Palgrave Mac Millan. ISBN: 97801137271945

Kraus, Sidney (2000). Televised presidential debates and public policy. New Jersey: Laurence Erlbaum Associates. ISBN: 9780805816020

López-García, Guillermo; Llorca-Abad, Germán; Valera-Ordaz, Lidia; Peris-Blanes, Alvar (2018). "Los debates electorales, ¿el último reducto frente la mediatización? Un estudio de caso de las elecciones generales españolas de 2015 ". Palabra clave, v. 21, n. 3, pp. 772-797.

https://palabraclave.unisabana.edu.co/index.php/palabraclave/article/view/7794

Lovenduski, Joni (2005). Feminizing politics. Cambridge, UK: Polity Press. ISBN: 9780745624631

Lozares, Carlos (1996). "La teoría de las redes sociales". Papers. Revista de sociología, v. 48, pp. $103-112$. https://doi.org/10.5565/rev/papers/v48n0.1814

Luengo, Óscar G. (2011). "Debates electorales en televisión: una aproximación preliminar a sus efectos inmediatos”. Revista española de ciencia política, n. 25, pp. 81-96.

https://recyt.fecyt.es/index.php/recp/article/view/37516

Luengo, Óscar G.; García-Marín, Javier (2009). “Liderazgo y debates electorales”. En: IX Congreso español de ciencia política. Málaga: Aecpa.

Martínez-Pandiani, Gustavo (2006). "El impacto de la televisión en la comunicación política moderna". Signos universitarios: Revista de la Universidad del Salvador, v. 25, n. 1, pp. 67-88.

https://p3.usal.edu.ar/index.php/signos/article/view/2955/3582

Mazzoleni, Gianpietro (2010). La comunicación política. Madrid: Alianza Editorial. ISBN: 9788420688862

McGregor, Shannon C.; Mourão, Rachel R. (2016). "Talking politics on Twitter: Gender, elections, and social network". Social media + society, v. 2, n. 3.

https://doi.org/10.1177/2056305116664218 
McKinney, Mitchell; Carlin, Diana (2004). Political campaign debates. New Jersey: Laurence Erlbaum Associates. ISBN: 9781410602787

Mead, George-Herbert (1934). Mind, self, and society: From the standpoint of a social behaviourist. Chicago: University of Chicago Press. ISBN: 9780226516677

Meeks, Lindsay (2013). A woman's place: Gender politics and Twitter in the 2012 elections. University of Washington: Washington.

https://digital.lib.washington.edu/researchworks/handle/1773/25186

Muñoz-Alonso, Alejandro (1999). Democracia mediática y campañas electorales. Barcelona: Ariel. ISBN: 978843441 2774

Newman, Mark E. J.; Girvan, Michelle (2004). "Finding and evaluating community structure in networks". Physical review $E$, v. 69, n. 2, 026113.

https://doi.org/10.1103/PhysRevE.69.026113

Norris, Pipa (1997). Women, media and politics. Oxford University Press: Oxford, UK. ISBN: 9780195105674

Orozco-Gómez, Guillermo (2014). "La televisión, lo televisivo y sus audiencias. El estallido de sus vínculos con la ficción”. Telos: Cuadernos de comunicación e innovación, n. 99, pp. 13-23.

https://telos.fundaciontelefonica.com/archivo/numero099/el-estallido-de-sus-vinculos-con-la-ficcion

Pindado, Fernando; Rebollo, Óscar (2015). El debate público. Informe sobre el debate público y calidad democrática. Barcelona: Escola de I'IGOP; Universitat Autònoma de Barcelona. https://igop.uab.cat/wp-content/uploads/2015/04/DEBATE_PUBLICO_2015_A4-IGOP.pdf

Quintas-Froufe, Natalia; González-Neira, Ana (2014). “Audiencias activas: participación de la audiencia social en la televisión". Comunicar, v. 22, n. 43, pp. 83-90.

https://doi.org/10.3916/C43-2014-08

Ross, Karen; Evans, Elizabeth; Harrison, Lisa; Shears, Mary; Wadia, Khursheed (2013). "The gender of news and news of gender: A study of sex, politics, and press coverage of the 2010 British general election". The international journal of press/politics, v. 18, n. 1, pp. 3-20.

https://doi.org/10.1177/1940161212457814

Saavedra-Llamas, Marta; Rodríguez-Fernández, Leticia (2018). “Las cadenas de televisión españolas frente al debate del 13J: estrategias de programación y audiencia social”. Fonseca, journal of communication, n. 17, pp. 125-136. https://doi.org/10.14201/fjc201817125136

Sánchez, José M. (2011). "Twitter mostró la 'otra' cara del debate”. ABC, 8 noviembre. https://www.abc.es/elecciones/abci-Twitter-debate-rubalcaba-rajoy-201111070000_noticia.html

Sartori, Giovanni (1998). Homo videns. La sociedad teledirigida. Buenos Aires: Taurus. ISBN: 9788430604692

Simmel, George (1908). On individuality and social forms. Chicago: University of Chicago Press. ISBN: 9780226757766

The Cocktail Analysis (2012). Observatorio de redes sociales. IV oleada. Madrid. https://es.slideshare.net/TCAnalysis/4-oleada-observatorio-de-redes-sociales

Tremayne, Mark; Minooie, Milad (2015). “Using social media to analyze candidate performance during televised political debates". Electronic news, v. 9, n. 3, pp. 143-159.

https://doi.org/10.1177/1931243115593321

Vergeer, Maurice; Franses, Philip-Hans (2016). "Live audience responses to live televised election debates: Time series analysis of issue salience and party salience on audience behavior". Information, communication \& society, v. 19, n. 10, pp. 1390-1410.

https://doi.org/10.1080/1369118X.2015.1093526

White, Harrison C. (1992). Identity and control: A structural theory as social action. Princeton: Princeton University Press. ISBN: 9780691003986 